\title{
Frontières
}

\section{Lettre à une petite fille qui, consolant sa poupée, ne savait pas qu'elle berçait la rumeur du monde}

\section{Fernande Goulet}

Volume 13, numéro 1, automne 2000

La mort au tableau noir

URI : https://id.erudit.org/iderudit/1074253ar

DOI : https://doi.org/10.7202/1074253ar

Aller au sommaire du numéro

Éditeur(s)

Université du Québec à Montréal

ISSN

1180-3479 (imprimé)

1916-0976 (numérique)

Découvrir la revue

Citer ce document

Goulet, F. (2000). Lettre à une petite fille qui, consolant sa poupée, ne savait pas qu'elle berçait la rumeur du monde. Frontières, 13(1), 68-69.

https://doi.org/10.7202/1074253ar d'utilisation que vous pouvez consulter en ligne. 


\section{LETTRE À UNE PETITE FILLE QUI, CONSOLANT SA POUPÉE, NE SAVAIT PAS QU'ELLE BERÇAIT LA RUMEUR DU MONDE}

Fernande Goulet

Lola ma petite,

Ce soir, je me sens bien loin dans l'espace d'une vieillesse qui s'impose à la manière d'une grande fatigue. Je viens vers toi, Lola, à ce moment précis où le temps me presse et où le compte de mes jours me laisse entrevoir la ligne d'horizon. Cette image d'un horizon lointain me rappelle les décors flamboyants, à perte de vue, derrière la grange du grand-père. Tu te souviens ? Par beau temps, chaque soir, il y avait quelque chose comme une noce à l'autre bout des champs. Que de fois, toi et moi avons contemplé, au loin tout au fond, à la limite des terres cultivables cette magie d'une lumière qui bougeait et descendait jusqu'à ne plus être vue. Nous avions la certitude que rien d'autre ne pouvait exister au-delà de cet immense faisceau de lumières, si ce n'était quelque chose d'innommable. Peut-être bien une sorte de paradis.

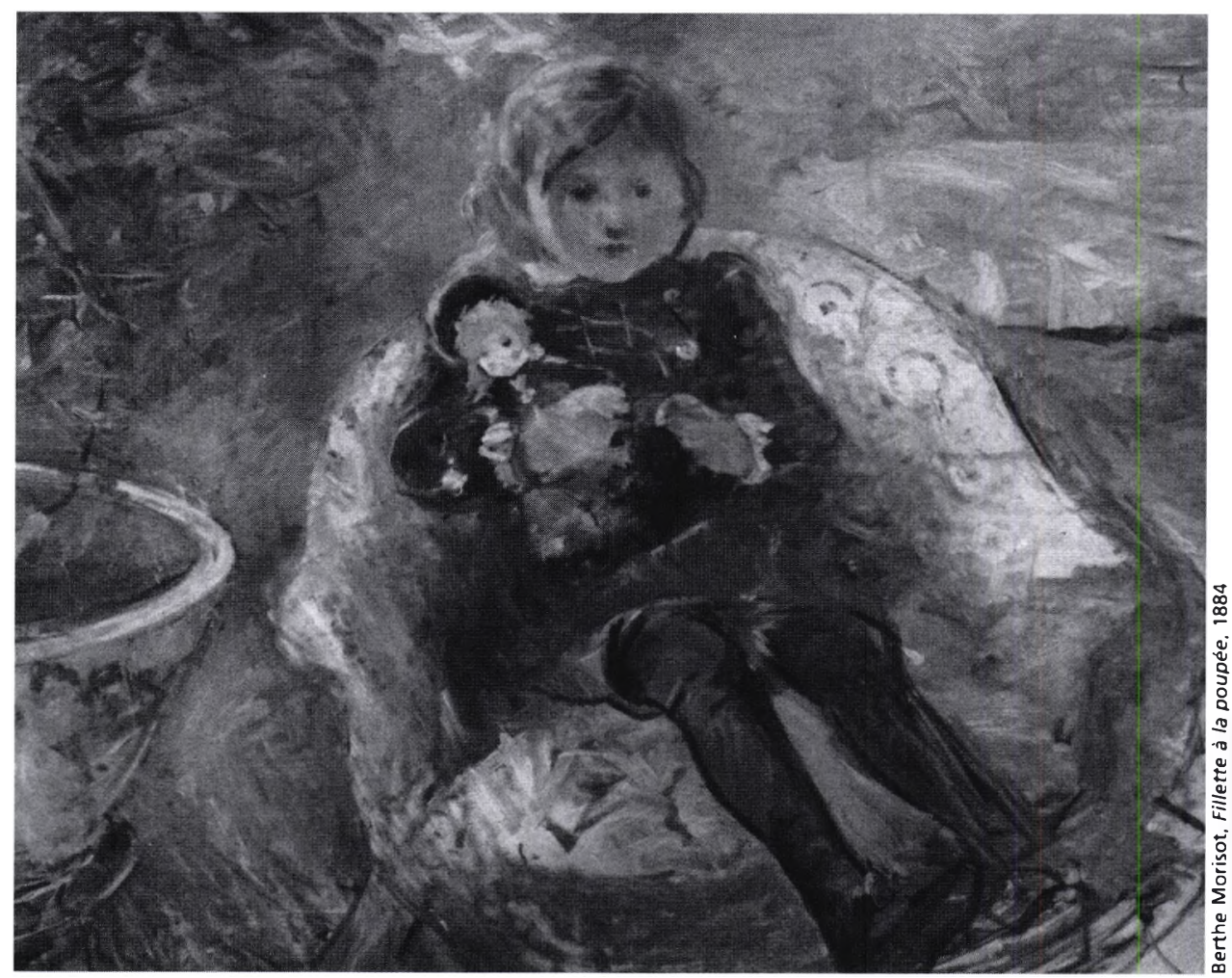


Tu sais, Lola, il m'a fallu beaucoup de jours pour m'approcher de ce qui pourrait être le terme de mon voyage. Chaque matin, habitée tantôt par le doute, tantôt par l'espoir tenace d'y arriver, j'ai dû reprendre le même sentier. Le mien. Durant ce long parcours, j'ai apprivoisé le bonheur d'inventer la vie, j'ai jonglé avec l'éphémère et me suis laissée glisser amoureusement dans le rythme du temps. Mais comme toi, Lola, j'ai été parfois hantée par la peur des ombres et des fantômes qui s'agitaient en des circonstances les plus imprévisibles. Alors, à folle allure, je fuyais sur ma route comme si la perspective du bonheur devenait pour moi tout aussi redoutable que celle du malheur.

Un jour, Lola, une petite fille comme toi mit sa mère dans l'embarras quand elle lui posa une question incroyable : "Dis, maman, c'est quand la vie ? " Cette petite, elle devait en avoir plein la tête de ces questions étonnantes! Moi, je te dirais que la vie que nous connaissons toi et moi, c'est maintenant. C'est tout de suite. Pour que tu comprennes bien, je dirais que la vie c'est comme un parfum qui remplit l'espace. La vie, elle flotte, elle respire en toi, elle court sans jamais nous dire d'où elle vient ni où elle va. Parfois elle rit au fond de nous et d'autres fois elle pleure. C'est elle qui fait surgir les crocus au printemps. C'est elle aussi qui fait bondir la chatte sur mes genoux et qui fait tenir aux branches des arbres les oiseaux qui dorment debout sur leurs pattes. La vie, c'est une grande énergie qui bouge tout le temps. Jamais elle ne se repose. C'est parce qu'elle est toujours en mouvement qu'on n'a jamais fini de la comprendre. Parfois généreuse et enivrante, on peut dire aussi qu'elle est, à certains moments, éprouvante et fuyante. Et c'est souvent quand la mort survient autour de soi qu'on se fâche contre la vie. On dit qu'elle est injuste et cruelle. Étrangement, on ne dit pas que la vie est méchante quant l'hiver fait place au printemps. Et pourtant, c'est pareil, toutes choses ayant un commencement et une fin. Les savants disent que c'est dans l'ordre des choses. La vie est ainsi faite qu'elle n'existerait pas si elle ne faisait pas la boucle avec la mort à la manière d'une élégante spirale vers le haut. Elle n'existerait pas si elle n'était pas propulsée dans sa dynamique par la mort.

La mort, quand elle arrive, fait d'immenses chagrins autour d'elle. Quand ton amie Bérangère est décédée à l'hôpital, c'est que la vie l'avait doucement quittée pour aller ailleurs. Parce que la vie va toujours ailleurs. Comme le coucher de soleil qui se laissait descendre en arrière de la montagne. Peut-être bien que la vie de Bérangère est allée rejoindre un quartier de lune ou un bel enfant qui avait du mal à venir au monde.

La mort, tu sais, ce n'est pas triste au point de ne pouvoir jamais se consoler. Mais il faut parfois beaucoup de temps pour oublier que le petit chat a déserté la maison ou que le serin, qui chantait si bien dans la salle à manger, ayant pris froid a été retrouvé mort dans le fond de sa cage. Mais perdre ses amis ou ses parents, ça fait des chagrins bien plus immenses. Aussi quand cela survient, il faut beaucoup parler de sa peine car tu sais, lorsqu'on pleure ou qu'on raconte son désarroi, ça fait de la place au dedans de soi pour la vie, et, tout doucement, ça console. Alors, on se souvient du coucher de soleil et de l'horizon lumineux qui se cachait derrière la grange du grand-père.

Mais Lola, il faut que je te dise qu'il y a un grand mystère dans la vie et la mort. Tu m'apprenais l'autre jour que ton professeur de catéchèse disait qu'il y a des mystères partout et que le plus gros des mystères c'est le Bon Dieu. Il n'avait pas tout à fait tort quand il disait cela ton professeur. Encore ne faut-il pas trop se hâter de mettre des mystères partout sans avoir beaucoup réfléchi, car il arrive qu'en grandissant on découvre des réponses à certaines questions qu'on se posait au temps où l'on était petit. Mais pour le Bon Dieu, c'est vrai que c'est un bien grand mystère. Mais un mystère, c'est superbe. Un mystère c'est comme un conte qui ne finit jamais. C'est comme un conte dans lequel on peut mettre nos plus beaux rêves. On dit que c'est un mystère parce que personne n'a trouvé de réponse à nos questions sur le Bon Dieu. Alors on imagine qu'il est quelque part derrière les couchers de soleil, qu'il s'enroule dans la neige blanche, qu'il se cache dans le cœur des enfants et parfois qu'on le retrouve dans les églises, là où il y a du silence et des lumières. Moi, je fais confiance au Bon Dieu et je me dis qu'il est partout où se trouve l'amour comme dans le cœur des enfants, dans les yeux des grands-mères, dans la musique de Mozart, dans les couchers de lune, dans la tendresse des mamans et la magie des papas qui soulèvent les enfants au bout de leurs bras. Le Bon Dieu, Il est là et c'est un bien beau mystère ! J'ose dire que la vie serait moins étonnante et infiniment moins merveilleuse s'il n'y avait pas l'incertitude et la fragilité des heures qui passent.
Petite Lola, je veux te dire que même si parfois la vie est contrariante et qu'elle met le cœur à l'épreuve, elle n'en fourmille pas moins de promesses et d'enchantement. Libre à toi d'y déposer les bons ingrédients. Parfois tu y arriveras. Parfois pas. Alors tu réinventeras la recette. Moi, quand je regarde ma vie avec une pointe d'humour, je pense qu'elle ressemble étrangement au bonsaï, cette plante têtue qui épouse les formes qu'on lui donne et qui audacieusement lance un sacré défi au temps fou de la modernité.

Ma petite Lola, cette lettre est bien sérieuse. Peut-être trop. Mais il y a tant de pensées qui tournent en rond au fond de moi. Peut-être, ne les as-tu entendues qu'à demi. Ce n'est pas grave. Dépose le tout dans ton coffre secret et si un jour tu avais le goût de repenser la vie, tu découvriras dans ce papier que le temps aura alors jauni, un testament. Celui d'une grand-mère qui te portait bien au chaud dans son cœur et qui, peut être bien, voulait auprès de toi, apprivoiser sa propre peur d'aller vers un " ailleurs " dont on ne sait rien mais qui pourrait bien être le matin...

\section{Moi qui t'aime}

Fernande GOULET s'est fait connaître notamment par son livre L'hibiscus était en fleurs, édité à Québec, aux éditions La Liberté, en 1990. 